\title{
Chemotherapy with Gemcitabine and Cisplatin for Advanced Ductal Adenocarcinoma of the Prostate: Clinical Courses of Two Patients
}

\author{
Yoshihiro Kamiyama, ${ }_{1}^{1}$ Koji Mitsuzuka, ${ }^{1}$ Mika Watanabe, ${ }^{2}$ Naoki Kawamorita, ${ }^{1}$ \\ Shigeyuki Yamada, ${ }^{1}$ Yasuhiro Kaiho, ${ }^{1}$ Akihiro Ito, ${ }^{1}$ Haruo Nakagawa ${ }^{1}$ and \\ Yoichi Arai ${ }^{1}$ \\ ${ }^{1}$ Department of Urology, Tohoku University Graduate School of Medicine, Sendai, Miyagi, Japan \\ ${ }^{2}$ Department of Pathology, Tohoku University Hospital, Sendai, Miyagi, Japan
}

\begin{abstract}
Ductal adenocarcinoma is an unusual variant of adenocarcinoma of the prostate with a poorly understood natural history, and its treatment is not well defined. Ductal adenocarcinoma is often diagnosed at an advanced stage, because no specific tumor markers are known. Docetaxel has been used for acinar adenocarcinoma of the prostate, a common type of prostate cancer, but it is largely ineffective for ductal adenocarcinoma. Earlier studies suggested that the chemotherapy with gemcitabine and cisplatin might be effective for ductal adenocarcinoma. Here we report two patients with ductal adenocarcinoma of the prostate that did not respond to docetaxel, but responded to the gemcitabine/cisplatin chemotherapy. Patient 1 was a 59-year-old man who had lung metastasis despite androgen deprivation therapy with undetectable levels of prostate-specific antigen and who presented with brain metastasis during docetaxel chemotherapy. Pathological examination of the resected brain tumor revealed ductal adenocarcinoma with positive immunostaining for carcinoembryonic antigen. The gemcitabine/cisplatin chemotherapy achieved partial response of the lung metastasis with serum carcinoembryonic antigen levels decreasing from 11.4 $\mathrm{ng} / \mathrm{mL}$ to $2.9 \mathrm{ng} / \mathrm{mL}$. Patient 2 was a 69-year-old man with lung metastasis. Local progression appeared during androgen deprivation therapy, and the subsequent transurethral biopsy revealed ductal adenocarcinoma with positive immunostaining for neuron-specific enolase. Bone and distant lymph node metastasis appeared despite docetaxel chemotherapy. Six courses of the gemcitabine/cisplatin chemotherapy achieved partial response of metastatic lesions, with serum neuron-specific enolase levels decreasing from $118 \mathrm{ng} / \mathrm{mL}$ to $2.6 \mathrm{ng} / \mathrm{mL}$. The gemcitabine/cisplatin chemotherapy is a potential option for treatment of advanced ductal adenocarcinoma of the prostate.
\end{abstract}

Keywords: chemotherapy; cisplatin; ductal adenocarcinoma; gemcitabine; prostate cancer

Tohoku J. Exp. Med., 2015 December, 237 (4), 317-321. (C) 2015 Tohoku University Medical Press

\section{Introduction}

Ductal adenocarcinoma of the prostate (ductal PCa) is rare and accounts for $0.4-0.8 \%$ of all prostate cancers (Wein 2002). It is a unique subtype of prostate cancer characterized by the presence of tall, pseudo-stratified, columnar cells with abundant cytoplasm arranged in a papillary pattern (Morgan et al. 2010). Serum prostate-specific antigen (PSA) levels are often lower than those of conventional acinar prostate cancer (acinar PCa), and it is often diagnosed at an advanced stage. Radical prostatectomy specimens of ductal PCa often show unfavorable histological features, including high Gleason grade, large tumor volume, high incidence of extraprostatic extension, seminal vesicle invasion, positive surgical margins, and lymph node metastasis, and its prognosis is poor (Christensen et al. 1991). Although surgical resection and radiation or intra-arterial or systemic chemotherapy have been reported as treatments for ductal PCa (Finamanti et al. 2011), a standard treatment strategy has not been established, especially for advanced ductal $\mathrm{PCa}$.

Docetaxel has been used as standard chemotherapy for advanced acinar PCa, but its efficacy for ductal PCa was not promising. The chemotherapy with gemcitabine and cisplatin (GC) has been used as standard first-line chemotherapy for urothelial carcinoma (von der Mase et al. 2005). Kato et al. (2007) reported that GC chemotherapy was effective for a patient with ductal PCa. Moreover, cisplatin-based chemotherapy was effective for variant types of prostate cancer including ductal PCa (Iguchi et al. 2003).

Received July 17, 2015; revised and accepted October 30, 2015. Published online December 3, 2015; doi: 10.1620/tjem.237.317.

Correspondence: Yoshihiro Kamiyama, Department of Urology, Tohoku University Graduate School of Medicine, 1-1 Seiryo-machi, Aoba-ku, Sendai, Miyagi 980-8574, Japan.

e-mail: souta0907@uro.med.tohoku.ac.jp 
Therefore, we performed GC chemotherapy for two patients with advanced ductal $\mathrm{PCa}$ and report the clinical courses for these two patients that did not respond to docetaxel.

\section{Case Presentation}

\section{Patient 1}

A 59-year-old man visited to previous office urology because of an abnormal PSA level on medical examination $(430 \mathrm{ng} / \mathrm{mL})$. Pathological examination of the prostate biopsy showed acinar $\mathrm{PCa}$ with Gleason score $4+5$ (cT4N1M1, lymph node and bone metastasis). Subsequently, he was referred to our hospital. Serum carcinoembryonic

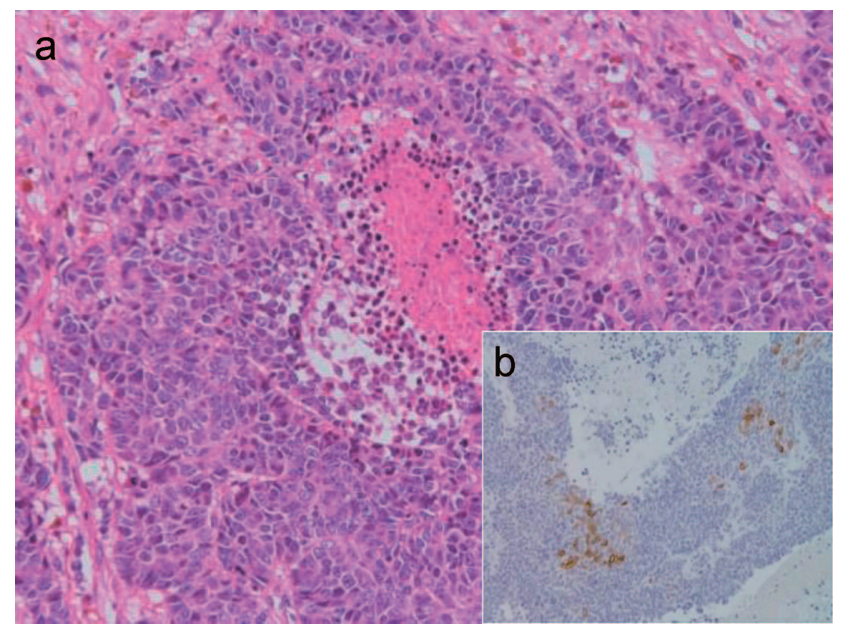

Fig. 1. Morphological analysis of the metastatic brain tumor excised from Patient 1.

(a) Hematoxylin-eosin staining. The presence of tall columnar ductal cancer cells with an increasing papillary pattern, with areas of comedo necrosis, indicates ductal adenocarcinoma $(\times 40)$. (b) Positive immunostaining for carcinoembryonic antigen $(\mathrm{CEA})(\times 200)$. antigen (CEA) was $4.6 \mathrm{ng} / \mathrm{mL}$, and serum carbohydrate antigen 19-9 (CA19-9) was $23.3 \mathrm{U} / \mathrm{ml}$, both of which were within normal ranges. Although androgen deprivation therapy (ADT) decreased the PSA to an undetectable level, perineal pain and urinary difficulty appeared. Transrectal prostate re-biopsy was done, which revealed mixed (acinar and ductal) adenocarcinoma. Computed tomography (CT) showed an enlarged prostate, and 74 Gy of external beam radiation were delivered to the prostate and seminal vesicles for local control. Although his urinary symptoms improved with ADT, lung metastasis appeared 35 months after initiation of ADT with an increased serum CEA level of $5.1 \mathrm{ng} /$ $\mathrm{mL}$ and an undetectable level of PSA. Docetaxel chemotherapy started, but brain metastasis appeared after 3 courses, with serum CEA increasing to $11.4 \mathrm{ng} / \mathrm{mL}$. Pathological findings of the resected brain tumor revealed pure ductal adenocarcinoma with positive immunostaining for CEA and negative for PSA (Fig. 1). The remaining immunostaining profile was CA19-9 (-), p63 (-), cytokeratin 7 (CK7) (-), CK20 (+), and caudal-related homeobox (CDX)-2 (+). GC chemotherapy, which consisted of gemcitabine $\left(1,000 \mathrm{mg} / \mathrm{m}^{2}\right)$ on days 1,8 , and 15 and cisplatin $\left(70 \mathrm{mg} / \mathrm{m}^{2}\right)$ on day 2 , every 4 weeks, started with the patient's agreement. This protocol was approved by the chemotherapy committee of the Tohoku University Hospital. Partial response (PR) was achieved for the lung metastatic lesions, with the serum CEA level decreasing to $2.9 \mathrm{ng} / \mathrm{mL}$ after 4 courses of GC chemotherapy. The disease progressed after 7 courses of GC chemotherapy and he died of the cancer 6 months later after termination of GC chemotherapy (Fig. 2).

\section{Patient 2}

A 69-year-old man was referred to our department

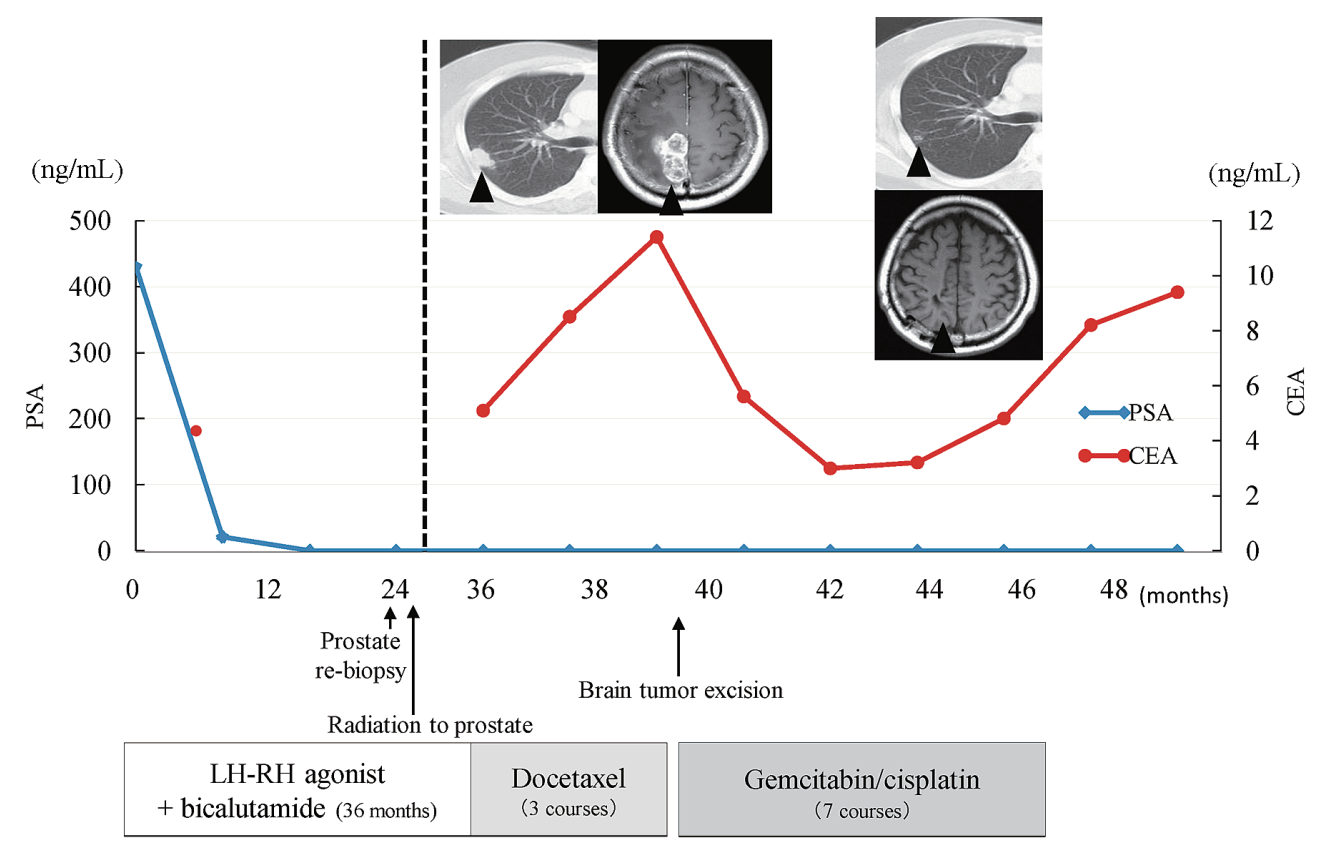

Fig. 2. Clinical course of Patient 1 with the changes of tumor markers. 
because of multiple lung tumors of unknown origin. Serum PSA level was $5.623 \mathrm{ng} / \mathrm{mL}$ with normal ranges of other tumor markers, including CEA, CA19-9 and neuron-specific enolase (NSE). A prostate biopsy showed acinar PCa with Gleason score 4+4 (cT3N0M1). Although ADT decreased the PSA to $0.156 \mathrm{ng} / \mathrm{mL}$, and the lung tumors shrank significantly, he complained of urinary pain and difficulty. CT showed an enlarged prostate protruding to the bladder, and the subsequent transurethral biopsy showed ductal PCa with focal positive PSA and positive NSE (Fig.

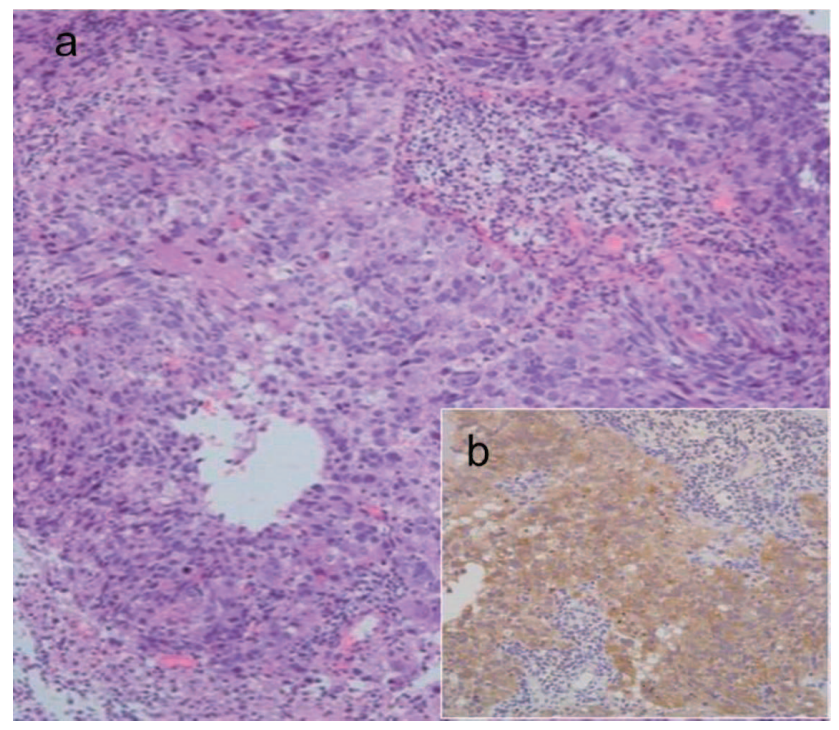

Fig. 3. Morphological analysis of the transurethral biopsy specimen from Patient 2.

(a) Hematoxylin-eosin staining. The morphological features indicate ductal adenocarcinoma $(\times 40)$, as seen Patient 1. (b) Positive immunostaining for neuron-specific enolase (NSE) $(\times 200)$.
$3)$. The remaining immunostaining profile was CEA $(+/-)$, CA 19-9 (+/-), p63 (-), CK7 (+), CK20 (+), and CDX2 (-). Although 74 Gy of external beam radiation for local control were delivered to the prostate and seminal vesicles with continuing ADT, serum NSE and PSA levels increased gradually, and CT showed lymphadenopathy of the mediastinum and multiple bone metastasis. Subsequently, docetaxel chemotherapy started, but new cervical spine metastasis and a listless feeling occurred in the patient after 7 courses; 44 Gy of external beam radiation were delivered for local control. Due to our previous experience, GC chemotherapy started with the patient's agreement and PR was achieved for lung and lymph node metastatic lesions, while bone metastasis were retained stable disease. After 6 courses of GC chemotherapy, serum PSA and NSE levels decreased from $12.148 \mathrm{ng} / \mathrm{mL}$ and $118 \mathrm{ng} / \mathrm{mL}$ to $0.016 \mathrm{ng} /$ $\mathrm{mL}$ and $2.6 \mathrm{ng} / \mathrm{mL}$, respectively (Fig. 4). Although lung and lymph node metastatic lesions shrank with ADT, brain metastasis appeared with increased levels of PSA and NSE. The patient asked for best supportive care and died of the cancer 6 months later after termination of GC chemotherapy.

\section{Discussion}

Ductal PCa has been recognized as an aggressive and variant type of prostate cancer that is different from usual acinar PCa (Wein 2002). The present two patients were first diagnosed as acinar $\mathrm{PCa}$ and responded to ADT, but then presented with local progression or distant metastasis. Secondary pathological examination of recurrent sites revealed ductal PCa. Although the major component of the tumor was initially acinar $\mathrm{PCa}$ that responded to ADT, a small amount of ductal PCa that was resistant to ADT might have become dominant during ADT (Yamashita et al.

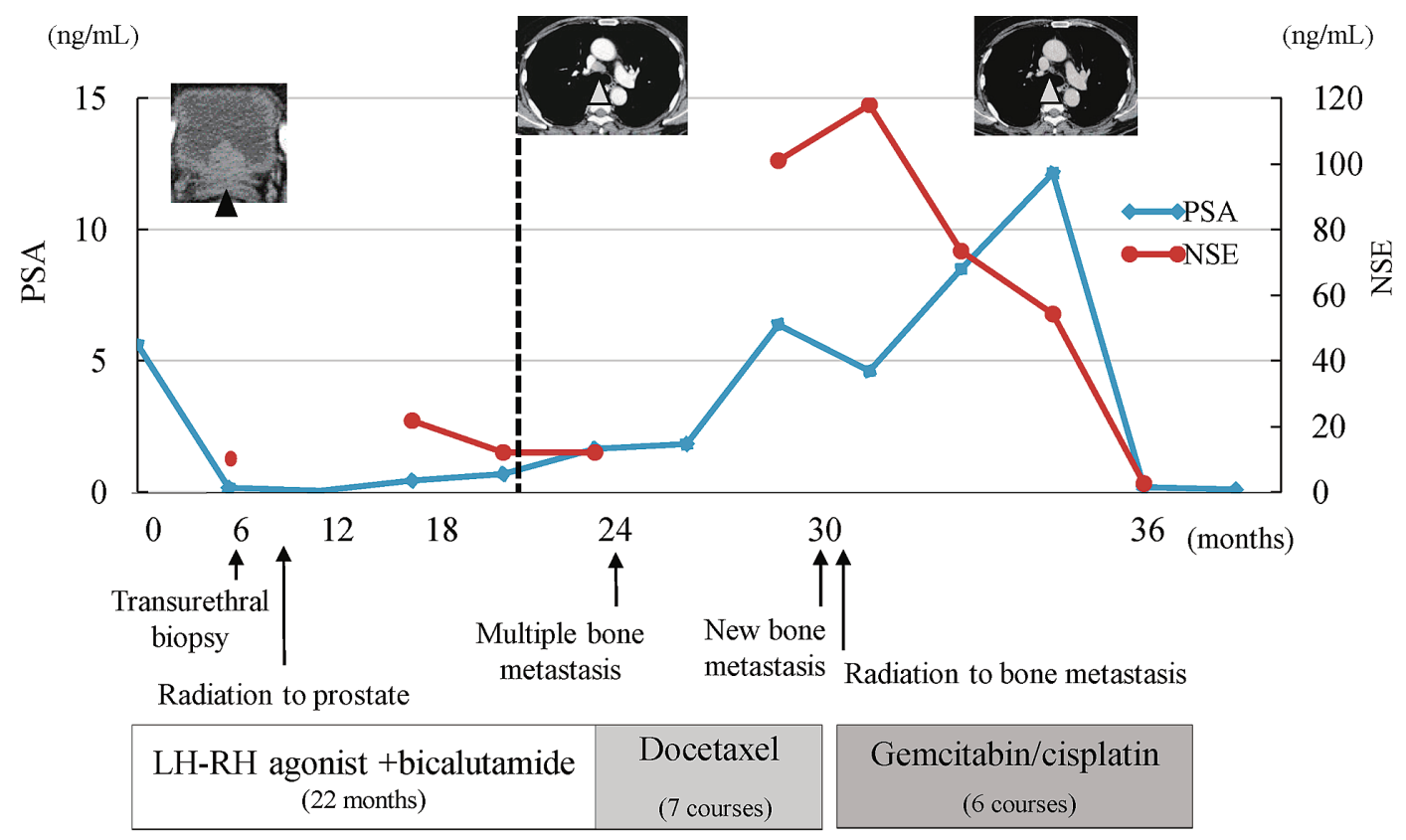

Fig. 4. Clinical course of Patient 2 with the changes of tumor markers. 
2008).

Standard chemotherapy for advanced ductal PCa remains unknown, and the reasons for the difficulty in determining the appropriate systemic chemotherapy for ductal PCa may derive from its variety or uncertainty in diagnosis. Although the diagnosis of ductal PCa is usually based on morphological findings, Seipel et al. (2014) reported interobserver variability regarding the diagnostic criteria and a wide variation of immunostaining patterns. A recent report studying the immunostaining patterns of ductal $\mathrm{PCa}$ demonstrated the difficulty of finding typical discriminating immunostaining patterns in ductal PCa (Jardel et al. 2013).

On the other hand, tumor markers other than PSA or detailed immunostaining findings in each case might be useful to know the tumor characteristics and to consider systemic treatment for metastatic ductal PCa. It is generally considered that no specific tumor markers are produced in ductal PCa, but CEA (Patient 1) and NSE (Patient 2) were elevated with tumor progression. Kato et al. (2007) reported a ductal PCa case with elevated serum CA19-9 levels that responded to GC chemotherapy. Recently, Bilen et al. (2014) reported that some patients with elevated carbohydrate antigen 125 (CA125) levels had tumors compatible with ductal PCa. Although the type or frequency of tumor marker elevation in ductal PCa has not been well studied, measurement of tumor markers might be useful to understand the disease condition or response to treatment in some cases of ductal PCa.

The immunostaining pattern of Patient 1 was similar to that of gastrointestinal cancer. Kato et al. (2007) used GC chemotherapy for ductal PCa with elevated serum CA19-9 levels, because CA19-9 was a typical tumor marker for pancreatic tumor for which GC chemotherapy was efficacious. Cisplatin was also used for gastric cancer (Koizumi et al. 2008), and we selected GC chemotherapy for Patient 1, while another treatment for gastrointestinal cancer including S-1 or molecular targeted agents might be an alternative to cisplatin. Aparicio et al. (2013) showed the effectiveness of cisplatin-based chemotherapy in variant types of prostate cancer, including small cell cancer. We selected GC chemotherapy after docetaxel failure because the immunostaining pattern of Patient 2 was supposed to have variant or small cell cancer features. In Patient 2, elevation of serum NSE indicated the possible presence of a neuroendocrine component. Although the morphological pattern of Patient 2 was considered to be ductal adenocarcinoma because of the tall, columnar cells with abundant cytoplasm arranged in a papillary pattern, a neuroendocrine tumor might show a ductal shape. Unfortunately, it was difficult to confirm the final diagnosis because of the small amount of sample from the transurethral biopsy, but the equivocal diagnosis of ductal carcinoma, which was based only on morphological structure, is a problem to be discussed in the future. The exact reasons why GC chemotherapy was effective for these ductal $\mathrm{PCa}$ patients remain unclear, but detailed immunostaining information would give us a chance to consider systemic chemotherapy for ductal PCa.

In conclusion, GC chemotherapy may be effective in certain patients with ductal $\mathrm{PCa}$, in which some tumor markers and detailed immunostaining results could be useful to consider systemic chemotherapy or to document the response to treatment. Further accumulation of evidence should be needed to determine systemic chemotherapy for advanced ductal PCa.

\section{Conflict of Interest}

The authors declare no conflict of interest.

\section{References}

Aparicio, A.M., Harzstark, A.L., Corn, P.G., Wen, S., Araujo, J.C., Tu, S.M., Pagliaro, L.C., Kim, J., Millikan, R.E., Ryan, C., Tannir, N.M., Zurita, A.J., Mathew, P., Arap, W., Troncoso, P., et al. (2013) Platinum-based chemotherapy for variant castrate-resistant prostate cancer. Clin. Cancer Res., 19, 36213630.

Bilen, M.A., Reyes, A., Bhowmick, D., Maa, A., Bast, R. Jr., Pisters, L.L., Lin, S.H., Logothetis, C.J. \& Tu, S.M. (2014) Variant prostate carcinoma and elevated serum CA-125. Can. J. Urol., 21, 7442-7448.

Christensen, W.N., Steinberg, G., Walsh, P.C. \& Epstein, J.I. (1991) Prostatic duct adenocarcinoma. Findings at radical prostatectomy. Cancer, 67, 2118-2124.

Finamanti, M., Antonelli, A., Contessa, P., Cosciani Cunico, S. \& Simeone, C. (2011) Ductal carcinoma of the prostate: impact on survival and therapeutic controversies of a rare tumor. Urologia, 78, 283-287.

Iguchi, T., Uchida, J., Nakamura, T., Kawamura, M., Wakasa, K. \& Nakatani, T. (2003) Effective combined chemotherapy for prostatic ductal adenocarcinoma: continuous venous infusion of 5-fluorouracil and low-dose consecutive cisplatin. BJU Int., 92 Suppl 3, e54-e55.

Jardel, P., Debiais, C., Godet, J., Irani, J. \& Fromont, G. (2013) Ductal carcinoma of the prostate shows a different immunophenotype from high grade acinar cancer. Histopathology, 63, 57-63.

Kato, T., Hashimoto, Y., Okada, S., Tozawa, K., Takahashi, S. \& Kohri, K. (2007) Carbohydrate antigen 19-9-positive prostatic ductal adenocarcinoma effectively treated with cisplatin and gemcitabine. Int. J. Urol., 14, 1103-1106.

Koizumi, W., Narahara, H., Hara, T., Takagane, A., Akiya, T., Takagi, M., Miyashita, K., Nishizaki, T., Kobayashi, O., Takiyama, W., Toh, Y., Nagaie, T., Takagi, S., Yamamura, Y., Yanaoka, K., et al. (2008) S-1 plus cisplatin versus S-1 alone for first-line treatment of advanced gastric cancer (SPIRITS trial): a phase III trial. Lancet Oncol., 9, 215-221.

Morgan, T.M., Welty, C.J., Vakar-Lopez, F., Lin, D.W. \& Wright, J.L. (2010) Ductal adenocarcinoma of the prostate: increased mortality risk and decreased serum prostate specific antigen. J. Urol., 184, 2303-2307.

Seipel, A.H., Delahunt, B., Samaratunga, H., Amin, M., Barton, J., Berney, D.M., Billis, A., Cheng, L., Comperat, E., Evans, A., Fine, S.W., Grignon, D., Humphrey, P.A., Magi-Galluzzi, C., Montironi, R., et al. (2014) Diagnostic criteria for ductal adenocarcinoma of the prostate: interobserver variability among 20 expert uropathologists. Histopathology, 65, 216-227.

von der Maase, H., Sengelov, L., Roberts, J.T., Ricci, S., Dogliotti, L., Oliver, T., Moore, M.J., Zimmermann, A. \& Arning, M. (2005) Long-term survival results of a randomized trial 
comparing gemcitabine plus cisplatin, with methotrexate, vinblastine, doxorubicin, plus cisplatin in patients with bladder cancer. J. Clin. Oncol., 23, 4602-4608.

Wein, A.J. (2002) Subtypes of prostate carcinoma. Campbell's Urology, 8th ed., edited by Walsh, P.C., et al. Saunders
Company, Philadelphia, pp. 2880

Yamashita, R., Matsuzaki, M., Matsui, T., Yamaguchi, R., Yuen, K., Niwakawa, M. \& Tobisu, K. (2008) Clinical characteristics of prostatic adenocarcinoma with ductal features. Nihon Hinyokika Gakkai Zasshi, 99, 525-530. 